\title{
Convexity of Interval-valued Fuzzy Sets Applied to Decision-Making Problems
}

\author{
Pedro Huidobro $^{a, c}$ and Pedro Alonso ${ }^{b}$ and Vladimir Janišs ${ }^{c}$ and Susana Montes ${ }^{a}$ \\ ${ }^{a}$ Dept. of Statistics and O.R., University of Oviedo, Oviedo, Spain, \{huidobropedro, montes\}@uniovi.es \\ ${ }^{b}$ Dept. of Mathematics, University of Oviedo, Oviedo, Spain, palonso@uniovi.es \\ ${ }^{c}$ Dept. of Mathematics, Matej Bel University, Banská Bystrica, Slovakia, vladimir . janis@umb.sk
}

\begin{abstract}
Fuzzy sets can help us with imprecision, however, there are situations we are not able to assign an accurate value. Interval-valued fuzzy sets are applied in decision-making to deal with imprecision. The convexity of interval-valued fuzzy sets establishes some interesting results about decision local and global maximizers.
\end{abstract}

Keywords: Interval-valued fuzzy sets, convexity, decision-making.

\section{Introduction}

In a decision-making process there are at least three relevant elements to keep in mind, 1) a set of alternatives, 2) a set of constraints on the option within several alternatives, and 3) a utility function that connects the gain or loss resulting from the election of that alternative with each decision. In many real situations, it is too difficult to specify accurately the objective function and the constraints. In order to work with imprecision, fuzzy sets can be a very useful tool [7]. Since Zadeh [20] introduced them, several extensions have been studied. The one we are considering is the family of interval-valued fuzzy sets (IVFS), which were introduced independently by Zadeh [21], Grattan-Guiness [10], Jahn [12] and Sambuc [16] in the seventies.

On the other hand, convexity is a mathematical notion that can be a useful tool in many different problems and, in particular, in decision-making problems. Since Zadeh introduced convexity for fuzzy sets, several authors have been developing this theory (see, for instance, Ammar and Metz [1], Diaz et al. [8], Ramik and Vlach [15], Syau and Lee [17] and Yang [19]). It has important applications in areas such as optimization [13].
Taking into account the previous comments, the main aim of this paper is to introduce a theory for applying convex interval-valued fuzzy sets to deal with decisionmaking and optimization problems, when the imprecision can be represented by means of this kind of sets.

This paper is organized as follows. In Section 2, some basic concepts are introduced and notations are fixed. Section 3 is devoted to propose a method for using interval-valued fuzzy sets to decision-making problems. In Section 4 we remark the importance of the concept of convexity for this kind of problems. Finally, some conclusions are drawn in Section 5.

\section{Basic concepts}

Let $X$ denote the universe of discourse. A fuzzy subset $A$ of $X$ is characterized by a function $A: X \rightarrow[0,1]$. The collection of all the fuzzy sets in $X$ is denoted by $F S(X)$. One of the fuzzy set extensions is the collection of interval valued fuzzy sets (IVFS). An IVFS on $X$ is defined by a function $A: X \rightarrow L([0,1])$ such that $A(x)=[\underline{A}(x), \bar{A}(x)]$, where $L([0,1])$ expresses the family of closed intervals contained in the unit interval $[0,1]$. Thus, an IVFS $A$ is described by two functions, $\underline{A}$ and $\bar{A}$, from $X$ into $[0,1]$ such that $\underline{A}(x) \leq \bar{A}(x)$, for all $\bar{x} \in X$. If $\underline{A}(x)=\bar{A}(x)$, for all $x \in X$, then $A$ is a classical fuzzy set. Let us denote by $I V F S(X)$ the collection of all the IVFS in $X$. A usual notation for describing the membership degrees of an IVFS at any point of the universe is the following:

$$
A=\{\langle x, A(x)\rangle: x \in X\}
$$

Since there is not a unique order to compare intervals, all the concepts for IVFSs are directly related to the chosen order on $L([0,1])$. One of the most usual order for intervals, which is induced by the usual partial order on $\mathbb{R}^{2}$, is the lattice order [9], defined as:

$$
a \preceq_{L o} b \text { if } \underline{a} \leq \underline{b} \text { and } \bar{a} \leq \bar{b}
$$


for any $a=[\underline{a}, \bar{a}]$ and $b=[\underline{b}, \bar{b}]$ in $L([0,1])$.

This relation in $L([0,1])$ is an order, but it is not a linear order. In order to solve this questions, Bustince et al. [5] introduced admissible orders, as a way to be coherent with this order but, at the same time, to deal with a linear order.

Definition 2.1 [5] Let $(L([0,1]), \preceq)$ be a poset. The order $\preceq$ is called an admissible order if

i) $\preceq$ is a linear order on $L([0,1])$,

ii) for all $[a, b],[c, d] \in L([0,1]),[a, b] \preceq[c, d]$ whenever $[a, b] \leq_{L o}[c, d]$.

An interesting property of admissible orders is that they can be built using aggregation functions [6].

Definition 2.2 $[2,14]$ Let $\mathscr{A}: \bigcup_{i=1}^{n}[0,1]^{n} \rightarrow[0,1]$ such that

- $\mathscr{A}(0,0, \ldots, 0)=0, \mathscr{A}(1,1, \ldots, 1)=1$,

- $\mathscr{A}$ is monotone in each variable,

then $\mathscr{A}$ is an aggregation function.

Aggregation functions are defined on $\bigcup_{i=1}^{n}[0,1]^{n}$, but we would like to work on $L([0,1])$. However, it is immediate that there is a natural bijection between $L([0,1])$ and $K([0,1])=\left\{(u, v) \in[0,1]^{2} \mid u \leq v\right\}$ which associates any interval $[\underline{a}, \bar{a}]$ in $L([0,1])$ to the point $(\underline{a}, \bar{a})$ in $[0,1]^{2}$ (see [5]). Thus, we can use aggregation functions to obtain just a value as a representative of the interval. Based on this idea, Bustince et al. construct the following method to make admissible orders.

Proposition 2.1 [5] Let $\mathscr{A}, \mathscr{B}:[0,1]^{2} \rightarrow[0,1]$ be two continuous aggregation functions, such that for all $(u, v),\left(u^{\prime}, v^{\prime}\right) \in K([0,1])$, the equalities $\mathscr{A}(u, v)=$ $\mathscr{A}\left(u^{\prime}, v^{\prime}\right)$ and $\mathscr{B}(u, v)=\mathscr{B}\left(u^{\prime}, v^{\prime}\right)$ can only hold if $(u, v)=\left(u^{\prime}, v^{\prime}\right)$. Define the relation $\preceq \mathscr{A}, \mathscr{B}$ on $L([0,1])$ by $a \preceq \mathscr{A}, \mathscr{B} b$ if and only if

$$
\mathscr{A}(\underline{a}, \bar{a})\langle\mathscr{A}(\underline{b}, \bar{b})
$$

or

$$
\mathscr{A}(\underline{a}, \bar{a})=\mathscr{A}(\underline{b}, \bar{b}) \text { and } \mathscr{B}(\underline{a}, \bar{a}) \leq \mathscr{B}(\underline{b}, \bar{b})) .
$$

Then $\preceq \mathscr{A}, \mathscr{B}$ is an admissible order on $L([0,1])$.

Two particular examples of admissible orders obtained by means of the previous proposition are the wellknown lexicographical orders (see [5]):

- Lexicographical order type 1: $a \preceq_{\text {Lex } 1} b$ if $\underline{a}<\underline{b}$ or $\underline{a}=\underline{b}$ and $\bar{a} \leq \bar{b}$.
- Lexicographical order type 2: $a \preceq_{\text {Lex2 }} b$ if $\bar{a}<\bar{b}$ or $\bar{a}=\bar{b}$ and $\underline{a} \leq \underline{b}$.

Given $A$ and $B$ two IVFSs and an order relation between intervals, denoted by $\preceq_{o}$, we say that $A$ is $o$ contained in $B$ iff $A(x) \preceq_{o} B(x)$ for all $x$ in $X$.

Taking in account this notion, Huidobro et al. [11] proposed a definition for the intersection of IVFS as the largest IVFS contained on them, which is the following:

Definition 2.3 Let $A, B$ be two interval-valued fuzzy sets in $X$ and let $\preceq_{o}$ be an order on $L([0,1])$. We define the $o$-intersection of $A$ and $B$, and we denote $A \cap_{o} B$ as the greatest interval-valued fuzzy set such that $A \cap_{o}$ $B \subseteq_{o} A$ and $A \cap_{o} B \subseteq_{o} B$.

As there is not a unique way to compare intervals, this definition changes depending on the chosen order on $\operatorname{IVFS}(X)$, as it was shown in [11]. In addition, if we use admissible orders, the existence of the intersection is ensured as admissible orders are linear orders too.

\section{Decision-making based on IVFSs}

In the literature, some theories can be found about using fuzzy sets in the field of decision-making. For instance, Bellman and Zadeh [3] pointed out that a decision could be seen as a group of goals and constraints with symmetry between these two concepts. This approach allow us to deal with goals and constrains as if they were notions which are connected in a symmetric manner by "and" connective.

In fuzzy set theory, it is accepted that we know the membership degree of the elements to the set. However, it often happens that the membership function is an imperfect information and it is not known precisely [4].There are situations where we are not sure about which is the proper value to assign to a fuzzy membership value, however, we can solve this problem by assigning an interval where the value is contained. Obviously, if we choose the same value for the endpoints, we are in the particular case of fuzzy sets.

We will continue using Bellman and Zadeh approach (see [3]), that is, if we consider the constraints and the goals as IVFS over the set of alternatives, $X$, then the decision $D$ would be the intersection of all the intervalvalued fuzzy constraints and goals.

In [18], Yager and Basson built a decision as the intersection of all the goals and the constrains. Taking into account this idea, we can obtain the following definition.

Definition 3.1 Let $X=\left\{x_{1}, \ldots, x_{n}\right\}$ be the set of al- 
ternatives, $G_{1}, \ldots G_{p}$ be the set of goals that can be expressed as IVFSs on the space of alternatives, and $C_{1}, \ldots, C_{m}$ be the set of constraints that can also be expressed as IVFSs on the space of alternatives. Let $\preceq_{o}$ be an order on $L([0,1])$. The goals and constraints then combine to form a decision $D$, which is an IVFS resulting from the intersection of the goals and the constrains. Thus, $D=G_{1} \cap_{o}, \ldots \cap_{o} G_{p} \cap_{o} C_{1} \cap_{o} \ldots \cap_{o} C_{m}$.

The interpretation of $D(x)$ could be the degree to that the alternative $x$ satisfies the goals and constraints, for any $x \in X$. Once the decision is built, we have to select the best alternative.

It is immediate from this definition that $D$ directly depends on the chosen order $\preceq_{o}$ in $L([0,1])$, since the intersection is really an $o$-intersection. Thus, the decision $D$, build as the intersection of the goals and constraints, would change depending on the order we are using.

Let us show an example, which is based on the example shown in [18].

Example 3.1 A person has to choose to locate a new plant in one of three locations $x_{1}, x_{2}$ and $x_{3}$. He wants to select a location that minimizes real estate cost, $G$, and is located near supplies, $C_{1}$. Let $X=\left\{x_{1}, x_{2}, x_{3}\right\}$. In this case, there is imprecision in the data, so IVFS would be more proper sets than FS. Let's suppose that the membership functions of the interval-valued fuzzy goal $G$ is

$$
\left\{\left\langle x_{1},[0.2,0.7]\right\rangle,\left\langle x_{2},[0.6,0.7]\right\rangle,\left\langle x_{3},[0.4,0.8]\right\rangle\right\}
$$

and the membership function of the interval-valued fuzzy constraint $C_{1}$ is

$$
\left\{\left\langle x_{1},[0.5,0.6]\right\rangle,\left\langle x_{2},[0.5,0.9]\right\rangle,\left\langle x_{3},[0.3,0.6]\right\rangle\right\}
$$

If we consider lexicographical order type 1, we emphasize the lower endpoint of the interval. Then the membership functions of the interval-valued fuzzy decision $D_{\text {lex } 1}$ is:

$$
\left\{\left\langle x_{1},[0.2,0.7]\right\rangle,\left\langle x_{2},[0.5,0.9]\right\rangle,\left\langle x_{3},[0.3,0.6]\right\rangle\right\}
$$

and the optimal decision would be $x_{2}$, since it is the alternative with a maximum value of $D_{\text {lex } 1}$ with respect to the lexicographical order type 1.

However, if we use lexicographical order type 2, then he membership functions of the interval-valued fuzzy decision $D_{\text {lex } 2}$ is:

$$
\left\{\left\langle x_{1},[0.5,0.6]\right\rangle,\left\langle x_{2},[0.6,0.7]\right\rangle,\left\langle x_{3},[0.4,0.8]\right\rangle\right\}
$$

and the optimal decision changes to $x_{3}$.
After this easy example, we can see the importance of a good selection of the order on $L([0,1])$ we are considering.

In an interval-valued fuzzy decision, like the previous one, all the goals and constraints are interval-valued fuzzy sets over the same set of alternatives, but in some situations it can change. Using the extension principle we can avoid this situation.

Definition 3.2 (Extension principle) Let $\preceq_{o}$ be an order on $L([0,1])$. Any given function $f: X \rightarrow$ $Y$ induces two functions, $f: \operatorname{IVFS}(X) \rightarrow \operatorname{IVFS}(Y)$ and $f^{-1}: \operatorname{IVFS}(Y) \rightarrow \operatorname{IVFS}(X)$, which are defined by $[f(A)](y)=\sup _{x \mid y=f(x)} A(x)$ for all $A \in \operatorname{IVFS}(X)$, where sup denotes the supremum using the order $\preceq_{o}$ and $\left[f^{-1}(B)\right](x)=B(f(x))$ for all $B \in I V F S(Y)$.

With this technique, the situation where the intervalvalued fuzzy constraints or goals are defined in different spaces can be mapped into the same space. When we have an $n$-ary function which maps $X_{1} \times X_{2} \times \cdots \times$ $X_{n}$ to $Y$, we would assume that if $A \in \operatorname{IVFS}\left(X_{1} \times X_{2} \times\right.$ $\left.\cdots \times X_{n}\right)$, then $A\left(x_{1}, x_{2}, \ldots, x_{n}\right)=A\left(x_{1}\right) \cap_{o} A\left(x_{2}\right) \cap_{o}$ $\cdots \cap_{o} A\left(x_{n}\right)$.

Let us illustrate it by the following example.

Example 3.2 Suppose the same conditions as in Example 3.1, but now there is another space $Y$ meaning a set of former works developed by the potential financial directors, $Y=\left\{y_{1}, y_{2}, y_{3}, y_{4}\right\}$. We have some information about these former works: $y_{1}$ and $y_{2}$ were made by $x_{1}, y_{3}$ was supervised by $x_{2}$ and $y_{4}$ was produced by $x_{2}$ and $x_{3}$.

With this information we construct the following mappig:

$$
f: Y \rightarrow X
$$

defined by $f\left(y_{1}\right)=x_{1}, f\left(y_{2}\right)=x_{1}, f\left(y_{3}\right)=x_{2}$ and $f\left(y_{4}\right)=\left\{x_{2}, x_{3}\right\}$.

We also known a fuzzy constraint over $Y$ that measures the impact of each one of works defined by: $C_{2}(Y)=$ $\left\{\left\langle y_{1},[0.4,0.6]\right\rangle,\left\langle y_{2},[0.7,0.9]\right\rangle,\left\langle y_{3},[0.75,0.8]\right\rangle\right.$,

$\left.\left\langle y_{4},[0.6,0.9]\right\rangle\right\}$. It is denoted as $C_{2}(Y)$ in order to point out that it is an interval-valued fuzzy set over the space Y. Now we should apply the extension principle to have all the goals and constraints as interval-valued fuzzy sets over the same space. To apply the extension principle we should first decide which order are we taking into account, in this case, we would use lexicographical order type 1. For $x_{1}$, $\left[f\left(C_{2}\right)\right]\left(x_{1}\right)=\sup _{y \mid y=f(x)} C_{2}(x)=\sup _{y_{1}, y_{2}} C_{2}(x)=$ $\sup \left\{C_{2}\left(y_{1}\right), C_{2}\left(y_{2}\right)\right\}=[0.7,0.9]$. Analogously, $\left[f\left(C_{2}\right)\right]\left(x_{2}\right)=[0.75,0.8]$ and $\left[f\left(C_{2}\right)\right]\left(x_{3}\right)=[0.6,0.9]$. 


$$
\begin{aligned}
& \text { Consequently, } C_{2}(X)= \\
& \qquad\left\{\left\langle x_{1},[0.7,0.9]\right\rangle,\left\langle x_{2},[0.75,0.8]\right\rangle,\left\langle x_{3},[0.6,0.9]\right\rangle\right\} .
\end{aligned}
$$

Finally, the decision is

$$
D=G \cap C_{1} \cap C_{2}
$$

that is, the membership degrees for the different alternatives in $D$ are:

$$
\left\{\left\langle x_{1},[0.2,0.6]\right\rangle,\left\langle x_{2},[0.5,0.9]\right\rangle,\left\langle x_{3},[0.3,0.6]\right\rangle\right\}
$$

Thus, the optimal decision is still $x_{2}$.

There are situations where some parameter in the decision is conditional upon other space. To be able to deal with such situations, Yager and Basson introduced the concept of fuzzy conditional set in [18]. Taking into account these ideas, we obtain the following definition for interval-valued fuzzy sets.

Definition 3.3 An IVFS B $(y)$ in $X$ is conditional on $y$ if its membership function depends on y as a parameter. This dependence is denoted $B(x \mid y)$.

Thus, if we are working with two spaces, $X$ and $Y$, and $y \in Y$, when there exists an interval-valued fuzzy set $B(y)$ on $X$, if we consider $A \in I V F S(Y)$, then $A$ induces an IVFS $B$ in $X$ whose membership function is $B(x)=\sup _{y} \min \{A(y), B(x \mid y)\}$.

Example 3.3 Suppose the same conditions of Example 3.2. The company is forced to minimize the facility of employing workers. They would concentrate on the distance to the main office. Let $Y=\{\operatorname{Near}(N), \operatorname{Med}(M), \operatorname{Far}(F)\}$. This constrain is given by the IVFS $C_{3}(Y)=$ $\{\langle N,[0.8,1]\rangle,\langle M,[0.4,0.7]\rangle,\langle F,[0.1,0.3]\rangle\}$. The relation between the alternatives and the proximity to the main office is given by the following conditioned IVFSs: $\quad C_{3}(X \mid N)=\left\{\left\langle x_{1},[0.7,0.8]\right\rangle\right.$, $\left.\left\langle x_{2},[0.5,0.6]\right\rangle,\left\langle x_{3},[0.3,0.6]\right\rangle\right\}, \quad C_{3}(X \mid M)=$ $\left\{\left\langle x_{1},[0.5,0.6]\right\rangle,\left\langle x_{2},[0.5,0.7]\right\rangle,\left\langle x_{3},[0.6,0.9]\right\rangle\right\}$, and $C_{3}(X \mid F)=\left\{\left\langle x_{1},[0.3,0.7]\right\rangle,\left\langle x_{2},[0.4,0.6]\right\rangle\right.$, $\left.\left\langle x_{3},[0.3,0.7]\right\rangle\right\}$. Thus, we can construct the interval-valued fuzzy set facility of hiring workers: For $x_{1}, C_{3}\left(x_{1}\right)=\sup _{y} \min \left\{C_{3}(y), C_{3}\left(x_{1} \mid y\right)\right\}=$ $\sup \left\{\min \left\{C_{3}(N)=[0.8,1], C_{3}\left(x_{1} \mid N\right)=\right.\right.$ $[0.7,0.8]\}, \min \left\{C_{3}(M)=[0.4,0.7], C_{3}\left(x_{1} \mid M\right)=\right.$ $[0.5,0.6]\}, \min \left\{C_{3}(F)=[0.1,0.3], C_{3}\left(x_{1} \mid F\right)=\right.$ $[0.3,0.7]\}\}=\sup [0.7,0.8],[0.4,0.7],[0.1,0.3]=$ $[0.7,0.8]$. We have to repeat the same procedure for $x_{2}$ and $x_{3}$. Thus, we obtain that the interval-valued fuzzy set $C_{3}(X)$ is given by:

$$
\left\{\left\langle x_{1},[0.7,0.8]\right\rangle,\left\langle x_{2},[0.5,0.6]\right\rangle,\left\langle x_{3},[0.4,0.7]\right\rangle\right\} \text {. }
$$

Finally, the decision is $D=G \cap C_{1} \cap C_{2} \cap C_{3}$, that is, the decision if the interval-valued fuzzy set $D$ defined as:

$$
\left\{\left\langle x_{1},[0.2,0.6]\right\rangle,\left\langle x_{2},[0.5,0.6]\right\rangle,\left\langle x_{3},[0.3,0.6]\right\rangle\right\} .
$$

Thus, $x_{2}$ is again the optimal decision.

\section{Convexity of interval-valued fuzzy sets}

Convexity is a very important property, mainly when we consider some specific decision-making problems. We will show its importance when the information is provided by means of interval-valued fuzzy sets.

Along this work, we will consider the notion of a convex interval-valued fuzzy set proposed by Huidobro et al. in [11].

Definition 4.1 [11] Let $X$ be an ordered space and let $\preceq_{o}$ be an order on $L([0,1])$. An interval-valued fuzzy set $A$ on $X$ is said to be o-convex, if for each $x<y<z$ in $X$ the following inequalities are fulfilled:

$$
A(x) \preceq_{o} A(y) \text { or } A(z) \preceq_{o} A(y) .
$$

But when we consider convexity as a tool in decisionmaking, we need also the concept of strict convexity.

Definition 4.2 Let $X$ be an ordered space and let $\preceq_{o}$ be an order on $L([0,1])$. An interval-valued fuzzy set $A$ on $X$ is said to be o-strictly convex, iffor each $x<y<z$ in $X$ the following inequalities are fulfilled:

$$
A(x) \prec_{o} A(y) \text { or } A(z) \prec_{o} A(y)
$$

which means that

$$
A(x) \preceq_{o} A(y) \text { and } A(x) \neq A(y)
$$

or

$$
A(z) \preceq_{o} A(y) \text { and } A(z) \neq A(y) \text {. }
$$

Combining convexity with admissible orders, which are also total orders, allowed Huidobro et al. [11] to prove that the intersection of two convex IVFS using admissible orders is also a convex IVFS. We will complete this result with the strict case. Thus,

Proposition 4.1 Let $X$ be an ordered space and let $\preceq_{\mathscr{A}, \mathscr{B}}$ be an admissible order based on two aggregations functions $\mathscr{A}$ and $\mathscr{B}$. If $A, B \in I V F S(X)$ are $\mathscr{A}, \mathscr{B}$-convex (resp. $\mathscr{A}, \mathscr{B}$-strictly convex) then $A \cap \mathscr{A}, \mathscr{B} B$ is also $\mathscr{A}, \mathscr{B}$-convex (resp. $\mathscr{A}, \mathscr{B}$-strictly convex), whenever it is not empty. 
This theorem is important if we think about the goals and the constraints in a decision-making process. If they are convex, then their intersection is also convex. Once the decision is a convex interval-valued fuzzy set, we can apply the following theorem, which allow us to obtain some interesting results in optimization.

Theorem 4.1 Let $A$ be a convex IVFS over the ordered space $X$. Let $\preceq_{o}$ be an order on $L([0,1])$. If $x^{*} \in \operatorname{supp}(A)=\left\{x \in X:[0,0] \prec_{o} A(x)\right\}$ is a strict local maximizer of $A(x)$, then it is also a global maximizer of $A(x)$ over supp $(A)$. The set of points at which $A(x)$ attains its global maximum over its support is a crisp convex set.

If we have a strictly convex IVFS instead of just a convex IVFS we can obtain better results. If we have strictly convexity, then the local maximizer is also a global maximizer while we need a strict local maximizer to assure that when we have convexity (not strict). Another interesting point is that if a strictly convex IVFS attains its maximum, it is unique.

Theorem 4.2 Let A be a strictly convex IVFS over the ordered space $X$. Let $\preceq_{o}$ be an order on $L([0,1])$.

i) If $x^{*} \in \operatorname{supp}(A)$ is a local maximizer of $A(x)$, then it is also a global maximizer.

ii) $A(x)$ attains its maximum over $\operatorname{supp}(A)$ at no more than one point.

It is time to combine a decision-making problem with the previous theorems:

Corollary 4.1 Let $\preceq_{o}$ be an order on $L([0,1])$, let $G_{1}, \ldots, G_{p}$ be the interval-valued fuzzy goals, $C_{1}, \ldots, C_{m}$ the interval-valued fuzzy constraints, and $D=G_{1} \cap, \ldots \cap G_{p} \cap C_{1} \cap, \ldots \cap C_{m}$ be the resulting decision.

- If the interval-valued fuzzy goals and the intervalvalued fuzzy constraints are convex IVFS, then the resulting decision D is a convex IVFS and the set of maximizing decisions of the IVFS D is a convex crisp set.

- If the interval-valued fuzzy goals and the intervalvalued fuzzy constraints are strictly convex IVFS, then the resulting decision $D$ is a strictly convex IVFS and the set of maximizing decisions of $D$ is a singleton or an empty set.

Let us summarize the decision-making problem of Example 3.3 in the following example.
Example 4.1 In the previous examples we consider one interval-valued fuzzy goal $G=$

$$
\left\{\left\langle x_{1},[0.2,0.7]\right\rangle,\left\langle x_{2},[0.6,0.7]\right\rangle,\left\langle x_{3},[0.4,0.8]\right\rangle\right\}
$$

and three interval-valued fuzzy constraints $C_{1}=$

$$
\left\{\left\langle x_{1},[0.5,0.6]\right\rangle,\left\langle x_{2},[0.5,0.9]\right\rangle,\left\langle x_{3},[0.3,0.6]\right\rangle\right\},
$$

$C_{2}=$

$$
\left\{\left\langle x_{1},[0.7,0.9]\right\rangle,\left\langle x_{2},[0.75,0.8]\right\rangle,\left\langle x_{3},[0.6,0.9]\right\rangle\right\}
$$

and $C_{3}=$

$$
\left\{\left\langle x_{1},[0.7,0.8]\right\rangle,\left\langle x_{2},[0.5,0.6]\right\rangle,\left\langle x_{3},[0.4,0.7]\right\rangle\right\} .
$$

If we suppose $x_{1}<x_{2}<x_{3}$, it is clear that $G, C_{1}, C_{2}$ and $C_{3}$ are strictly convex IVFS with respect to the lexicographical order type 1 , so the decision $D$ is also a convex IVFS w.r.t. the same order. It is easy to check it, since $D=$

$$
\left\{\left\langle x_{1},[0.2,0.7]\right\rangle,\left\langle x_{2},[0.5,0.6]\right\rangle,\left\langle x_{3},[0.3,0.6]\right\rangle\right\} .
$$

We can apply the previous result to assert that $x_{2}$ is a global maximizer.

As changing the order could be also interesting, in the following example we show what happens if we use lexicographical order type 2 .

Example 4.2 Using the same IVFS for the goal and contraints from the previous example, the decisionmaking problem is $G=$

$$
\left\{\left\langle x_{1},[0.2,0.7]\right\rangle,\left\langle x_{2},[0.6,0.7]\right\rangle,\left\langle x_{3},[0.4,0.8]\right\rangle\right\},
$$

$C_{1}=$

$$
\left\{\left\langle x_{1},[0.5,0.6]\right\rangle,\left\langle x_{2},[0.5,0.9]\right\rangle,\left\langle x_{3},[0.3,0.6]\right\rangle\right\},
$$

$C_{2}=$

$$
\left\{\left\langle x_{1},[0.7,0.9]\right\rangle,\left\langle x_{2},[0.6,0.9]\right\rangle,\left\langle x_{3},[0.6,0.9]\right\rangle\right\}
$$

and $C_{3}=$

$$
\left\{\left\langle x_{1},[0.7,0.8]\right\rangle,\left\langle x_{2},[0.4,0.7]\right\rangle,\left\langle x_{3},[0.4,0.7]\right\rangle\right\} .
$$

It should be noticed that there are changes in the constrains $C_{2}$ and $C_{3}$ because we used lexicographical order type 2 and it affects to the supremum and the minimum. Moreover, the constraints $C_{2}$ and $C_{3}$ are convex IVFS while $G$ and $C_{1}$ are stryctly convex IVFS. We can also see that $D$ is a convex IVFS, as $D=$

$$
\left\{\left\langle x_{1},[0.5,0.6]\right\rangle,\left\langle x_{2},[0.4,0.7]\right\rangle,\left\langle x_{3},[0.3,0.6]\right\rangle\right\} .
$$

Thus, $D$ is not only convex but strictly convex, so we can assure that $x_{1}$ is the unique optimal decision. 


\section{Concluding remarks}

In this paper, we propose a method for using intervalvalued fuzzy sets and their convexity to decisionmaking or optimization problems. It must be noticed that the subjectivity of IVFSs when assigning membership functions to the sets may help to construct convex interval-valued goals and constraints. That could be very useful as we were able to prove that a local maximizer could be easily a global maximizer. We also would like to point out that the order used to compare intervals is really relevant in order to obtain an optimal decision.

\section{Acknowledgement}

Authors would like to thank for the support of the grant no. 1/0150/21 provided by Slovak grant agency VEGA (V. Janiš), Spanish Ministry of Science and Technology project TIN-2017-87600-P (P. Alonso), Spanish Ministry of Science and Technology project PGC2018098623-B-I00 (P. Huidobro and S. Montes), FICYT Project IDI/2018/000176 (P. Alonso, P. Huidobro and S. Montes) and the Severo Ochoa program BP19-169 (P. Huidobro).

\section{References}

[1] E. Ammar and J. Metz. On fuzzy convexity and parametric fuzzy optimization. Fuzzy Sets and Systems, 49(2):135 - 141, 1992.

[2] G. Beliakov, H. Bustince, and T. Calvo. A Practical Guide to Averaging Functions. Springer, 2016.

[3] R. E. Bellman and L. A. Zadeh. Decision-making in a fuzzy environment. Management Science, 17(4):B-141-B-164, 1970.

[4] H. Bustince. Interval-valued fuzzy sets in soft computing. International Journal of Computational Intelligence Systems, 3(2):215 - 222, 2010.

[5] H. Bustince, J. Fernandez, A. Kolesárová, and R. Mesiar. Generation of linear orders for intervals by means of aggregation functions. Fuzzy Sets and Systems, 220:69 - 77, 2013.

[6] H. Bustince, C. Marco-Detchart, J. Fernandez, C. Wagner, J.M. Garibaldi, and Z. Takáč. Similarity between interval-valued fuzzy sets taking into account the width of the intervals and admissible orders. Fuzzy Sets and Systems, 390:23 47, 2020.

[7] Ernest Czogała and Hans-Jürgen Zimmermann. Decision making in uncertain environments.
European Journal of Operational Research, 23(2):202 - 212, 1986.

[8] S. Díaz, E. Induráin, V. Janiš, J. V. Llinares, and S. Montes. Generalized convexities related to aggregation operators of fuzzy sets. Kybernetika, $53: 383-393,2017$.

[9] J.A Goguen. L-fuzzy sets. Journal of Mathematical Analysis and Applications, 18(1):145 - 174, 1967.

[10] I. Grattan-Guinness. Fuzzy membership mapped onto intervals and many-valued quantities. Mathematical Logic Quarterly, 22(1):149 - 160, 1976.

[11] P. Huidobro, P. Alonso, V. Janiš, and S. Montes. Orders preserving convexity under intersections for interval-valued fuzzy sets. In MarieJeanne Lesot, Susana Vieira, Marek Z. Reformat, João Paulo Carvalho, Anna Wilbik, Bernadette Bouchon-Meunier, and Ronald R. Yager, editors, Information Processing and Management of Uncertainty in Knowledge-Based Systems, pages 493 - 505, Cham, 2020. Springer International Publishing.

[12] K.U. Jahn. Intervall-wertige mengen. Mathematische Nachrichten, 68:115 - 132, 1975.

[13] L. Liberti. Reformulation and convex relaxation techniques for global optimization. Quarterly Journal of the Belgian, French and Italian Operations Research Societies, 2(3):255 - 258, 2004.

[14] Radko Mesiar and Magda Komorníková. Aggregation functions on bounded posets. In Chris Cornelis, Glad Deschrijver, Mike Nachtegael, Steven Schockaert, and Yun Shi, editors, 35 Years of Fuzzy Set Theory: Celebratory Volume Dedicated to the Retirement of Etienne E. Kerre, pages 3 - 17, Berlin, Heidelberg, 2011. Springer.

[15] J. Ramik and M Vlach. Generalized Concavity in Fuzzy Optimization and Decision Analysis. Kluwer Academic Publishers, 2002.

[16] R. Sambuc. Function phi-flous, application a l'aide au diagnostic en pathologie thyroidienne. These de Doctorat en Medicine, 1975.

[17] Y. Syau and E Lee. Fuzzy convexity and multiobjective convex optimization problems. Computers \& Mathematics with Applications, 52(3):351 - 362, 2006.

[18] R. Yager and D. Basson. Decision making with fuzzy sets. Decision Sciences, 6(3):590-600, 1975. 
[19] X. Yang. A property on convex fuzzy sets. Fuzzy Sets and Systems, 126:269 - 271, 2002.

[20] L.A. Zadeh. Fuzzy sets. Information and Control, 8(3):338 - 353, 1965.

[21] L.A. Zadeh. The concept of a linguistic variable and its application to approximate reasoning-I. Information Sciences, 8(3):199 - 249, 1975. 